\title{
Review \\ Idiopathic pulmonary fibrosis: an epithelial/fibroblastic cross-talk disorder
}

\author{
Moisés Selman and Annie Pardo
}

Instituto Nacional de Enfermedades Respiratorias \& Facultad de Ciencias, UNAM, México DF, México

Correspondence: Moisés Selman, Instituto Nacional de Enfermedades Respiratorias, Tlalpan 4502; Col. Sección XVI, México DF, CP 14080 , México. Tel: +525665 0043; fax: +525665 4623; e-mail: mselman@conacyt.mx

Received: 26 July 2001

Revisions requested: 17 August 2001

Revisions received: 20 August 2001

Accepted: 21 August 2001

Published: 11 October 2001
Respir Res 2002, 3:3

(C) 2002 BioMed Central Ltd

(Print ISSN 1465-9921; Online ISSN 1465-993X)

\begin{abstract}
Idiopathic pulmonary fibrosis is a chronic and usually progressive lung disorder of unknown etiology. A growing body of evidence suggests that, in contrast to other interstitial lung diseases, IPF is a distinct entity in which inflammation is a secondary and non-relevant pathogenic partner. Evidence includes the presence of similar mild/moderate inflammation either in early or late disease, and the lack of response to potent anti-inflammatory therapy. Additionally, it is clear from experimental models and some human disease that it is possible to have fibrosis without inflammation. An evolving hypothesis proposes that IPF may result from epithelial micro-injuries and abnormal wound healing.
\end{abstract}

Keywords: apoptosis, epithelial cells, extracellular matrix, myofibroblasts, pulmonary fibrosis

\section{Introduction}

Idiopathic pulmonary fibrosis (IPF), also referred to as cryptogenic fibrosing alveolitis, is a chronic, progressive and usually lethal lung disorder of unknown etiology. The disease occurs predominantly from middle age onwards, with more than two-thirds of patients being over 60 years old at the time of presentation. Men are nearly twice as likely as women to suffer from IPF [1].

Similar to other interstitial lung diseases, IPF has been considered for a long time as the deleterious consequence of an unresolved chronic inflammatory process that follows an unrecognized insult. Examining our knowledge in perspective, however, there is little evidence to sustain this hypothesis.

It is certain that most interstitial lung diseases include an identifiable initial inflammatory response to an unknown (for example, sarcoidosis and desquamative interstitial pneumonia) or known (for example, hypersensitivity pneu- monitis and drugs) damaging agent. In all these disorders, lung biopsy in early disease shows pure inflammation and, on follow-up, many of these patients improve or heal with anti-inflammatory therapy. A number of these disorders may naturally evolve to fibrosis and thus can often be difficult to differentiate from an IPF lung; for example, patients in the late stages of chronic hypersensitivity pneumonitis also present severe fibrosis and honeycombing [2].

By contrast, no one (or almost no one) seems to have seen an early phase of a true IPF, and the histology of patients presenting with IPF, both at 6 months and at 2 years after the beginning of symptoms, shows variable degrees of fibrosis and honeycombing with mild to moderate inflammation. In other words, there is no evidence that IPF starts with an inflammatory process and, most importantly, there is no evidence, other than parallelisms with other interstitial lung disorders, to support the concept that inflammation is more prominent in the early stages of IPF. In addition, most patients with IPF do not

$\mathrm{IPF}=$ idiopathic pulmonary fibrosis; $\mathrm{MMP}=$ matrix metalloproteinases; PAI = plasminogen activator inhibitor; TGF = transforming growth factor; $\mathrm{TIMP}=$ tissue inhibitor of metalloproteinases; UIP = usual interstitial pneumonia. 
respond to anti-inflammatory drugs, and the use of corticosteroids with or without immunosuppressive drugs only helps in a few cases, and then only produces a transient improvement without beneficial effects in the long term. In fact, survival curves for IPF are worse than for many cancers, with a median survival of 3-4 years after diagnosis $[3,4]$.

It has been suggested that IPF is characterized by a sequence of events that start with alveolar epithelial microinjuries followed by the formation of fibroblastic foci and result in an exaggerated deposition of extracellular matrix, which drives the destruction of the lung parenchyma architecture $[5,6]$.

In this context, we propose that there are two routes (at least) for developing diffuse pulmonary fibrosis. One of these is the 'inflammatory route', which is represented by almost all the non-IPF interstitial lung diseases where there is an early, clearly distinguishable phase of alveolitis and a late fibrotic phase. The other is the 'epithelial/fibroblastic route', which is represented by IPF.

It is important to emphasize here that the bleomycin model, the most used experimental model of lung fibrosis, is a paradigm of the inflammatory route. Animals sacrificed at early stages (i.e., 3 days post-instillation) show virtually inflammation only, but after a time (i.e., 14-21 days post-instillation) they show both inflammation and fibrosis. Furthermore, it has been reported recently that in the chronic stages, at least in rats, the model does not behave as a restrictive syndrome and the main histological features consist of focal peribronchiolar inflammation and fibrosis associated with paracicatricial emphysematous changes [7].

Accordingly, there are no appropriate experimental models of IPF, not only because we do not know its etiology, but also because all the traditional models start with an inflammatory reaction.

\section{Some light emerging from the new histopathological classification of idiopathic interstitial pneumonias}

During the past decade, it became increasingly evident that the term 'idiopathic pulmonary fibrosis' was imprecisely applied to a heterogeneous group of several distinct forms of idiopathic interstitial pneumonias, including usual interstitial pneumonia (UIP), desquamative interstitial pneumonia, cryptogenic organizing pneumonia, and acute interstitial pneumonia. Moreover, it was also clear that the histopathological findings in a number of patients with idiopathic interstitial pneumonias did not fit into any defined pattern, and in 1994, Katzenstein and Fiorelli [6] introduced the concept of nonspecific interstitial pneumonia for such lung biopsies.
Now it is widely accepted that the definite diagnosis of IPF requires a compatible clinical history and a surgical lung biopsy showing histological features of UIP. The latter is essential because we know that while other idiopathic interstitial pneumonias may improve or even heal after treatment, IPF/UIP is habitually progressive. As mentioned for chronic hypersensitivity pneumonitis, however, patients with a fibrotic or mixed cellular/fibrotic pattern of nonspecific interstitial pneumonia may also display a poor outcome [8].

An important concept regarding the possible role of alveolitis in the pathogenesis of IPF arises from this new classification. A careful review of the UIP lung has demonstrated that the inflammatory component is usually mild, occurs mainly in areas of collagen deposition or honeycomb change, and rarely involves otherwise unaltered alveolar septa [9]. There is little evidence to support the concept that inflammation is a prominent feature at any stage of the disease and moreover, severe inflammation should lead the clinician/pathologist to consider a diagnosis other than UIP.

In this sense, it is remarkable that we have been unable to clearly define the early changes in IPF/UIP. Actually, early and late IPF/UIP histology is the same, with a temporal heterogeneous combination of normal alveoli, some inflammation, fibroblastic foci, extracellular matrix accumulation and honeycombing. It is likely that this characteristic variegated temporal appearance of UIP reflects the repetitive occurrence over time of microscopic foci of lung injury and repair. In addition, a recent review of the Denver experience demonstrated that neither inflammation nor fibrosis correlates with survival. Interestingly enough, the only pathological data that showed a significant correlation with mortality were numbers of areas with fibroblastic foci [10].

An unsolved question in IPF/UIP is when alveolitis shows up during the development of the disease; our contention is that inflammation may appear only after the formation of fibroblastic foci.

\section{Is it possible to have a fibrotic response in non-inflammatory processes?}

Explants of mouse lungs cultured in a free-blood environment at various stages after hyperoxic injury have demonstrated that as alveolar damage, particularly of type 1 pneumocytes, increases, epithelial cell proliferation slackens while fibroblast growth becomes predominant [11]. In these explants, an increase in collagen synthesis and deposition was also noticed. Importantly, when the hyperoxic injury involved only the endothelial cells, no association with fibrosis was observed. We can learn two lessons from this model. First, as the fibrotic changes occurred in the presence of few macrophages, and in the absence of inflammatory cells from the systemic circulation, these findings 
clearly suggest that it is possible to have a fibrotic reaction without inflammation. Second, epithelial cell damage and perturbation of epithelial-fibroblast interactions is sufficient to promote the development of a fibrotic process.

A non-inflammatory fibrotic response in humans is provoked by chronic overuse of tendons. The lesion is frequently called tendinitis. A common example is the tennis elbow or lateral epicondylitis. Histopathological studies have shown, however, that this lesion contains few inflammatory cells [12], and suggest that chronic refractory lateral epicondylitis is a degenerative rather than an inflammatory condition. This may account for its lack of response to anti-inflammatory medication. By contrast, tendonitis, which usually occurs after acute tendon injuries, is characterized by the presence of an increased number of lymphocytes and neutrophils. In this context, it has recently been proposed that tennis elbow lesion represents a fibroblastic and vascular response (angiofibroblastic degeneration) to repetitive microtrauma and is now more commonly known as tendinosis [13]. The histopathological features represent a non-inflammatory, degenerative process associated with fibroblastic hyperplasia and the accumulation of disorganized and immature collagen.

\section{What is IPF if not an inflammatory disorder?}

We propose that IPF is, from the earliest stage, an epithelial-fibroblastic disease, that is, a fibroproliferative disorder preceded by alveolar epithelial cell activation. It is becoming apparent that the primary sites of ongoing injury and repair are the regions of fibroblastic proliferation, so-called fibroblastic foci $[9,14]$. These small aggregates of actively proliferating fibroblasts/myofibroblasts constitute multiple, microscopic sites of ongoing alveolar epithelial injury and activation associated with evolving fibrosis (Fig. 1). In other words, we postulate that in IPF, an initial epithelial cell injury and activation induces fibroblast migration/proliferation and fibroblast phenotypic change (myofibroblasts). Subsequently, myofibroblasts may provoke basement membrane disruption and alveolar epithelial cell apoptosis, perpetuating the damage and avoiding appropriate re-epithelialization. The final result is the excessive deposition of extracellular matrix with the destruction of the alveolar-capillary units and the formation of cystic fibrotic spaces or honeycombing (Fig. 2).

\section{The alveolar epithelial cell connection}

IPF lungs exhibit noteworthy changes in alveolar epithelium. These include the presence of cuboidal cells (hyperplasia of type 2 pneumocytes), reactive large and elongated epithelial cells (transitional cells among type 2 and type 1 pneumocytes?), microscopic areas of epithelial cell dropout, and bronchiolar epithelium lining areas of honeycomb lesions. In general terms, it seems that in IPF/UIP, the ability of type 2 alveolar cells to restore damaged type 1 cells is seriously affected [15].
Figure 1

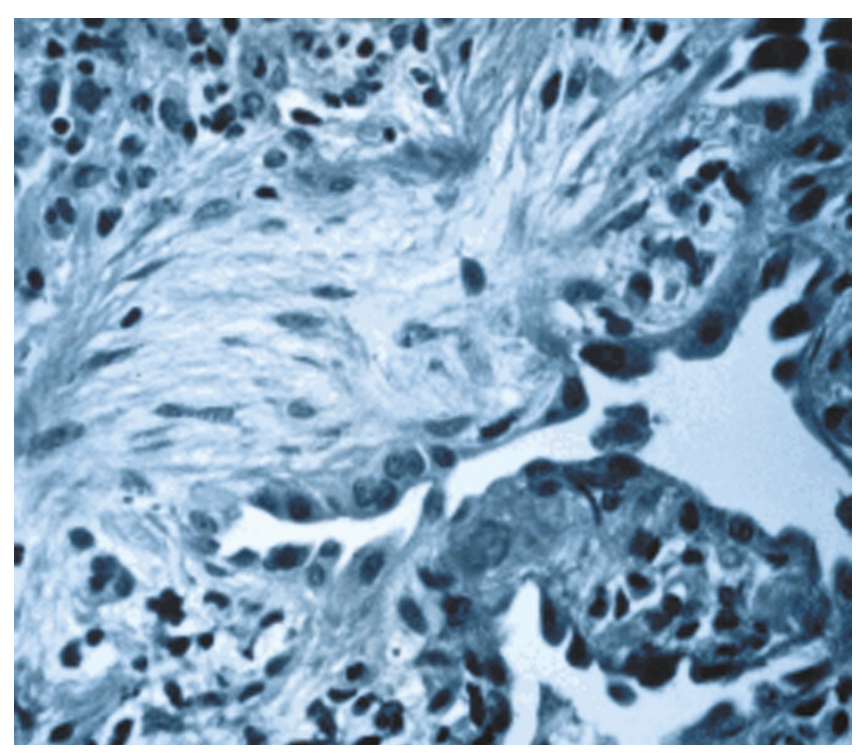

Subepithelial fibroblastic foci in an IPF lung. Notice the marked changes in alveolar epithelium (hematoxylin \& eosin, $\times 40$ original magnification).

Importantly, altered alveolar type 2 cells synthesize a variety of enzymes, such as matrix metalloproteinases, cytokines and growth factors, suggesting that the contribution of the epithelium in the extracellular matrix remodeling is greater than commonly thought. In fact, during the development of IPF, alveolar epithelial cells appear to be responsible for the expression and release of most, if not all, the profibrotic cytokines and growth factors that have usually been associated with inflammatory cells, primarily alveolar macrophages.

Thus, for example, Kapanci et al. [16] examined lung biopsy specimens from a number of IPF patients and demonstrated that type 2 alveolar epithelial cells constitute the main site of synthesis of transforming growth factor (TGF)- $\beta 1$ and tumor necrosis factor- $\alpha$. They also seemed to be closely related to the presence of myofibroblastic foci. Similar results were obtained by Nash et al. [17], who detected a widespread tumor necrosis factor- $\alpha$ staining of epithelial cells, particularly hyperplastic type 2 pneumocytes, while macrophages stained only weakly. Khalil et al. $[18,19]$ also found that in IPF, TGF- $\beta$ is located in alveolar macrophages and epithelial cells. In particular, epithelial cells of honeycomb cysts and hyperplastic type 2 pneumocytes stained intensely. TGF- $\beta$ was also localized in subepithelial regions of honeycomb cysts in areas of dense fibroconnective tissue deposition.

Other growth factors besides TGF- $\beta$ and tumor necrosis factor- $\alpha$ have been found to be expressed by epithelial 


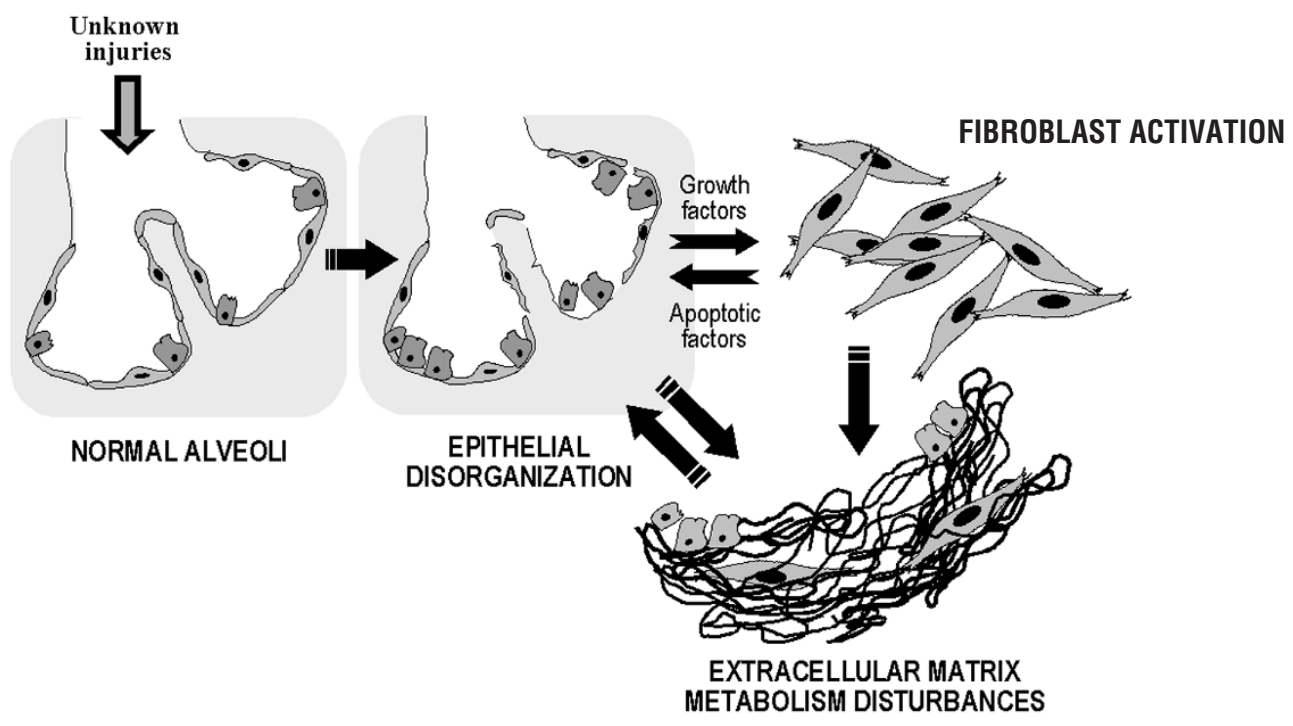

Hypothetical scheme of the main pathogenic events in IPF/UIP. Unknown insults provoke multiple microscopic foci of epithelial damage and stimulation. Activated alveolar epithelial cells release factors inducing fibroblast migration and proliferation and changes in cell phenotype. In the microenvironment of the lesion, myofibroblasts can induce epithelial cell apoptosis and basement membrane disruption, thus contributing to abnormal re-epithelialization and perpetuation of a vicious circle. Finally, fibroblasts/myofibroblasts secrete excessive amounts of extracellular matrix components and a disequilibrium between MMPs and TIMPs means that matrix degradation does not occur. The final result is an aberrant remodeling of the lung parenchyma.

cells in IPF. For example, we have found using immunofluorescence and in situ hybridization that the main cells expressing platelet-derived growth factor were alveolar epithelial cells [20]. However, the contribution of other cells, both inflammatory and resident, to the cytokine milieu during the development of IPF can not currently be ruled out.

\section{The fibroblast connection}

The earliest and probably the only morphological change associated with progression to fibrosis is the presence of fibroblastic foci (Fig. 1). A focus of fibroblasts is characterized by a distinct cluster of fibroblasts/myofibroblasts within the alveolar wall, with little if any associated inflammation within the foci. Both electron microscopy and immunohistochemistry have shown that fibroblast foci represent microscopic zones of acute lung injury in which alveolar lining cells are destroyed and the epithelial basement membrane is denuded $[14,21,22]$. The fibroblast proliferation occurs in an attempt to repair the damaged alveolus, and is followed by extracellular matrix accumulation. As mentioned, in these foci, scattered mononuclear inflammatory cells may accompany the process, but neither interstitial nor intraluminal inflammation are major features.

Fibroblastic foci may occur in the interstitial (subepithelial) space or in the alveolar spaces, which are later covered by reactive epithelium $[9,21,22]$. In the later stages of fibro- sis, epithelial basement membranes are frequently wavy and disrupted, and fibroblasts migrate through gaps in the epithelial basement membranes and continue proliferating and producing extracellular matrix in the alveolar spaces.

\section{Chronicle of a foretold programmed cell death: alveolar epithelial cells versus fibroblasts}

During normal skin wound healing, epithelial cells move quickly across the wound defect to re-establish the cutaneous cover. During the subsequent process of tissue repair, fibroblasts move into the wound space where they transform into myofibroblasts that contract the wound and construct new extracellular matrix. After that, programmed cell death has been proposed to mediate the decrease of mesenchymal cells. Finally, epidermal cells differentiate to re-establish the permeability barrier.

In a similar way, the complex process of normal lung repair after injury requires the collaborative efforts of several cell lineages and should include, on the one hand, epithelial cell migration, proliferation and differentiation, and on the other, fibroblast migration and proliferation, their transformation into myofibroblasts, and myofibroblast apoptosis. In progressive fibrotic disorders including IPF, however, current evidence suggests that the opposite situation occurs, such that increased and continuous epithelial cell apoptosis and decreased fibroblast/myofibroblast apoptosis take place. 
A number of studies performed both in vitro and in vivo support the notion that epithelial programmed cell death and necrosis are highly increased in IPF lungs. For example, we have demonstrated that altered fibroblasts emerging during fibrotic lung injury in IPF release angiotensin peptides and perhaps other soluble factors capable of inducing programmed cell death and net loss of alveolar epithelial cells $[23,24]$. Likewise, fibrotic human lungs exhibited increased in situ end labeling of fragmented DNA in the alveolar epithelium, which appears to be most concentrated immediately adjacent to underlying foci of myofibroblasts [25]. Both electron microscopy and picrosirius red confirmed the presence of epithelial cell apoptosis, necrosis and cell loss adjacent to foci of collagen accumulation surrounding fibroblast-like cells. More recently, it was reported that apoptosis of type II pneumocytes also occurred in the normal alveoli of most of IPF/UIP patients, whereas it was absent in the control lungs [26].

IPF lungs also exhibit upregulated Fas expression in bronchiolar and alveolar epithelial cells, suggesting that the Fas-Fas ligand pathway may also be involved [27]. Moreover, it was recently reported that the immunoreactivity for the Fas-associated death domain protein as well as for caspase- 1 and caspase- 3 were significantly increased in alveolar epithelial cells of IPF compared with controls [28]. Furthermore, Fas ligation induced upregulation of caspase- 1 and caspase- 3 expression in the nucleus and cytoplasm of A549 cells. These results strongly suggest that the Fas signalling pathway is upregulated in lung epithelial cells during IPF.

These findings together demonstrate that numerous alveolar epithelial cells die during the course of lung fibrosis, and support the hypothesis that alveolar epithelial cell death is at least partially induced by abnormal lung fibroblasts.

Keloids represent an abnormal wound healing response that fails to resolve and leads to formation of a raised collagen mass extending beyond the original wound margins that rarely regresses. The other type of excessive skin scarring is the hypertrophic scar, which, in contrast to keloids, is limited to the initial boundaries of the injury and tends to regress with time [29]. It was recently demonstrated that keloid-derived fibroblasts, in contrast to hypertrophic scarderived and normal skin-derived fibroblasts, are significantly resistant to both Fas-mediated and staurosporine-induced apoptosis [30]. Moreover, TGF- $\beta 1$, a prototype of fibrotic cytokines, appeared to have a pivotal role in this resistance to apoptosis [30]. Similarly, fibroblasts obtained from the superficial and basal regions of keloids showed population doubling times and saturation densities that were similar to those of age-matched normal fibroblasts [31]. In contrast, fibroblasts from the center of the keloid lesions showed a significantly reduced growth rate [31]. Although apoptotic cells were detected in both normal and keloid-derived fibroblasts, their numbers were two-fold lower in all keloid fibroblasts than in normal cells [31].

The in vivo apoptotic activity during IPF/UIP has also been clearly detected lower in the fibroblastic foci than in the fibromyxoid lesions of cryptogenic organizing pneumonia (a reversible disease), suggesting that fibroblasts/myofibroblasts in IPF survive for longer [32]. In contrast, we have recently found that fibroblasts from IPF lungs in culture have a lower growth rate than fibroblasts from normal lungs (the center of keloid lesions show a similarly reduced growth rate) and exhibit increased spontaneous apoptosis [33].

These in vitro findings may be explained in two ways. First, the microenvironment of IPF lungs may include anti-apoptotic factors that could influence fibroblast behavior in vivo but which are lost when cells are cultured in vitro. Second, it is possible that the different subpopulations selected for culture in vitro and which die by apoptosis are not those that are resistant to apoptosis in vivo. In this context, it has recently been reported that some experimental conditions assumed to occur in vivo may lead to the selection and propagation of certain apoptosis-resistant or apoptosis-susceptible fibroblast subpopulations [34]. Resistance to apoptosis occurs when cells are stimulated with TGF- $\beta 1$, and this subpopulation seems to represent an important part of the systemic sclerosis fibroblast phenotype [34].

Interestingly, some apparently contradictory results have been reported concerning lung fibroblast proliferation in vitro. Thus, some data suggest that human lung fibroblasts derived from fibrotic tissues proliferate significantly faster than those obtained from normal lungs [35]. The contradiction between these data and ours might be explained by the observation that higher rates of proliferation occur in fibroblasts derived from areas of early fibrosis, whereas lower rates of proliferation occur in cells obtained from dense fibroses [36].

\section{Coagulation cascade and fibrosis: a pathogenic role for epithelial cells and fibroblasts}

Lung injury leads to vasodilatation, with the subsequent leakage of plasma proteins into interstitial and alveolar spaces, activation of the coagulation cascade and deposition of fibrin. Fibroblasts migrate into this provisional fibrin matrix where they synthesize extracellular matrix molecules. The key protein involved in fibrin resorption is plasmin, a proteinase that also has a role in the activation of matrix metalloproteinases (MMPs), primarily MMP-1 and MMP-9 [37,38]. In turn, activation of MMPs provokes extracellular matrix degradation that, together with fibrin removal, results in the clearance of the alveolar spaces. Activated MMP-9 may also enhance epithelial cell migra- 
tion [37], and all of these constitute essential steps in the restoration of injured tissues to normal.

In contrast, the persistence of fibrin and inappropriate regulation of plasmin promotes fibrosis. Essentially, the balance between procoagulant (i.e. tissue factor), fibrinolytic (tissue-type and urokinase-type plasminogen activators) and anti-fibrinolytic (plasminogen activator inhibitor (PAI)-1 and PAI-2) systems determines whether fibrin will be deposited or resorbed.

In this context, increased local procoagulant and anti-fibrinolytic activities have been found in patients with IPF, suggesting that fibrin matrix removal is slow in them and, as a consequence, fibroblast migration, extracellular matrix deposition and the magnitude of the fibrotic response is increased $[39,40]$. The levels of tissue factor, PAl-1 and PAI-2 are significantly elevated in bronchoalveolar lavage obtained from IPF patients, and the compensatory increase in tissue factor pathway inhibitor appears to be insufficient to counterbalance tissue factor, which leads to a hypercoagulable state in IPF lungs [41].

Importantly for our hypothesis that IPF is an epithelial/ fibroblastic disorder, cuboidal alveolar epithelial cells appear to be the primary source of these activities. Tissue factor and PAl-1 and PAl-2 are thus both strongly expressed by alveolar epithelial cells in IPF lungs [39-41]. Furthermore, it has recently been found that whereas cryptogenic organizing pneumonia lesions have an increased expression of urokinase-type plasminogen activator, especially in the lining of epithelial cells around the fibroblastic plugs, the predominant staining in IPF lungs was for PAI-1, which was also in the epithelial cells lining the enlarged, restructured airspaces [42].

In addition, fibroblasts might also play a role in excessive fibrin deposit; it was found recently that dermal fibroblasts derived from keloid lesions markedly overexpress PAl-1 mRNA transcripts and protein compared to normal skin fibroblasts. If this finding can be extrapolated to lungs, it may suggest that lung fibroblasts could also participate in the synthesis of the major negative physiologic regulator of plasmin activation and, consequently, in the excessive alveolar fibrin accumulation [43].

\section{Progressive accumulation of extracellular matrix: a decisive role for fibroblasts/myofibroblasts}

The final result of IPF is an aberrant tissue remodeling, characterized by excessive deposition of connective tissue with the consequent progressive destruction of the lung parenchyma to form fibrotic honeycomb lesions. The molecular mechanisms behind this abnormal remodeling are poorly understood, but the loss of a regulated turnover involves, at least in part, two families of proteins: MMPs and the tissue inhibitors of metalloproteinases (TIMPs).
Recent evidence suggests that profound changes in MMP and TIMP expression and localization take place in the IPF lung microenvironment [44-46]. In general, these studies demonstrate an upregulation of collagenase-1 (MMP-1) and both gelatinases (MMP-2 and MMP-9), as well as the four TIMPs. Location is critical for appropriate function, however, and, interestingly, MMP-1 is usually seen in alveolar epithelial cells and alveolar macrophages, but hardly in fibroblasts or the extracellular matrix. In contrast, the inhibitors are strongly expressed and widely distributed throughout the injured IPF lung. In particular, TIMP-2 is predominantly expressed by myofibroblasts within the fibroblastic foci $[45,46]$. For this reason, it has been proposed that a non-degradative lung microenvironment prevails in IPF, which enhances the progressive accumulation of fibrillar collagens [46]. Moreover, fibroblasts derived from IPF lungs express in vitro significantly higher amounts of TIMPs than fibroblasts from normal lungs, without differences in collagenase-1 expression [33]. In addition, myofibroblasts represent an aggressive phenotype, being the main subset responsible for fibrillar collagen accumulation [47].

On the other hand, the epithelial expression of MMP-1 raises several questions about what this enzyme is doing there, relatively far away from where fibrilar collagens are being deposited. A putative MMP-1 function could be related to alveolar epithelial cell migration, as has been proposed for keratinocytes in skin wound healing. In fact, collagenase- 1 is prominently and invariably expressed by basal keratinocytes at the migratory front in all forms of cutaneous wounds [48].

A different situation may occur with the over-expression of gelatinase $A$ and gelatinase $B$. These MMPs have been localized, among other cellular sources, in subepithelial fibroblasts/myofibroblasts, occasionally in areas of denuded alveolar basement membranes, suggesting that they may participate in basement membrane disruption. This pathological process may, on the one hand, contribute to the failure of a correct re-epithelialization and, on the other, facilitate the migration of fibroblasts/myofibroblasts into the alveolar spaces.

\section{Conclusion}

The prevailing dogma regarding the pathogenesis of the fibrotic lung disorders take as a fact that there is an initial lung injury that is followed by an inflammatory process and then by fibroblast proliferation and fibrosis. This notion assumes that inflammation precedes and drives the fibrotic response and that it plays a major role in lung fibrogenesis.

This hypothetical concept appears to explain the pathogenesis of most interstitial lung diseases but not IPF/UIP, which seems to be an epithelial fibroblastic disease. Distinguishing fibrosis driven by inflammation from fibrosis 
originating as an epithelial/fibroblastic disorder is important not just for intellectual accuracy. Appropriate therapy relies on the correct understanding of the nature of the response to injury.

\section{Acknowledgement}

Programa Universitario de Investigación en Salud.

\section{References}

1. International consensus statement: Idiopathic pulmonary fibrosis: diagnosis and treatment. Am J Respir Crit Care Med 2000, 161:646-664.

2. Perez-Padilla R, Salas J, Chapela R, Sanchez M, Carrillo G, Perez $\mathrm{R}$, Gaxiola M, Selman M: Mortality in Mexican patients with chronic pigeon breeder's lung compared with those with usual interstitial pneumonia. Am Rev Respir Dis 1993, 148:4953

3. Bjoraker JA, Ryu JH, Edwin MK, Myers JL, Tazelaar HD, Schroeder DR, Offord KP: Prognostic significance of histopathologic subsets in idiopathic pulmonary fibrosis. Am J Respir Crit Care Med 1998, 157:199-203.

4. Douglas WW, Ryu JH, Schroeder DR: Idiopathic pulmonary fibrosis. Impact of oxygen and colchicine, prednisone, or no therapy on survival. Am J Respir Crit Care Med 2000, 161: 1172-1178.

5. Selman M, King TE, Pardo A: Idiopathic pulmonary fibrosis: prevaling and evolving hypotheses about its pathogenesis and implications for therapy. Ann Intern Med 2001, 134:136151.

6. Katzenstein ALA, Fiorelli RF: Nonspecific interstitial pneumonia/fibrosis. Histologic features and clinical significance. $A m \mathrm{~J}$ Surg Pathol 1994, 18:136-147.

7. Borzone G, Moreno R, Urrea R, Meneses M, Oyarzun M, Lisboa $\mathrm{C}$ : Bleomycin-induced chronic lung damage does not resemble human idiopathic pulmonary fibrosis. $A m J$ Respir Crit Care Med 2001, 163:1648-1653.

8. Nicholson AG, Colby TV, du Bois RM, Hansell DM, Wells AU: The prognostic significance of the histologic pattern of interstitial pneumonia in patients presenting with the clinical entity of cryptogenic fibrosing alveolitis. Am J Respir Crit Care Med 2000, 162:2213-2217.

9. Katzenstein ALA, Myers JL: Idiopathic pulmonary fibrosis. Clinical relevance of pathologic classification. Am J Respir Crit Care Med 1998, 157:1301-1315.

10. King TE, Sdchwarz MI, Brown K, Tose JA, Colby TV, Waldron JA, Flint A, Thurlbeck W, Cherniack RM: Extent of fibroblastic foci predict mortality in idiopathic pulmonary fibrosis. Am J Respir Crit Care Med 2001, 163:A982.

11. Adamson IYR, Young L, Bowden DH: Relationship of alveolar epithelial injury and repair to the induction of pulmonary fibrosis. Am J Pathol 1988, 130:377-383.

12. Regan W, Wold LE, Coonrad R, Morrey BF: Microscopic histopathology of chronic refractory lateral epicondylitis. Am J Sports Med 1992, 20:746-749.

13. Kraushaar BS, Nirschl RP: Tendinosis of the elbow (tennis elbow): clinical features and findings of histological, immunohistochemical, and electron microscopy studies. J Bone Joint Surg Am 1999, 81:259-279.

14. Kuhn C, III, Boldt J, King TE, Jr, Crouch E, Vartio T, McDonald JA: An immunohistochemical study of architectural remodeling and connective tissue synthesis in pulmonary fibrosis. Am Rev Respir Dis 1989, 140:1693-1703.

15. Kasper M, Haroske G: Alterations in the alveolar epithelium after injury leading to pulmonary fibrosis. Histol Histopathol 1996, 11:463-483.

16. Kapanci Y, Desmouliere A, Pache JC, Redard M, Gabbiani G: Cytoskeletal protein modulation in pulmonary alveolar myofibroblasts during idiopathic pulmonary fibrosis. Am J Respir Crit Care Med 1995, 152:2163-2169.

17. Nash JRG, McLaughlin PJ, Butcher D, Corrin B: Expression of tumour necrosis factor- $\alpha$ in cryptogenic fibrosing alveolitis. Histopathology 1993, 22:343-347.

18. Khalil N, O'Connor RN, Unruh HW, Warren PW, Flanders KC, Kemp A, Bereznay $\mathrm{OH}$, Greenberg $\mathrm{AH}$ : Increased production and immunohistochemical localization of transforming growth factor- $B$ in idiopathic pulmonary fibrosis. Am J Respir Cell Mol Biol 1991, 5:155-162.

19. Khalil N, O'Connor RN, Flanders KC, Unruh HW: TGF-B1, but not TGF-B2 or TGF-B3, is differentially present in epithelial cells of advanced pulmonary fibrosis: an immunohistochemical study. Am J Respir Cell Mol Biol 1996, 14:131-138.

20. Antoniades $\mathrm{NH}$, Bravo M, Avila R, Galanopoulus T, Neville J, Selman M: Platelet-derived growth factor in idiopathic pulmonary fibrosis. J Clin Invest 1990, 86:1055-1064.

21. Kuhn C, McDonald JA: The roles of the myofibroblast in idiopathic pulmonary fibrosis. Ultrastructural and immunohistochemical features of sites of active extracellular matrix synthesis. Am J Pathol 1991, 138:1257-1265.

22. Fukuda Y, Basset F, Ferrans VJ, Yamanaka N: Significance of early intra-alveolar fibrotic lesions and integrin expression in lung biopsy specimens from patients with idiopathic pulmonary fibrosis. Hum Pathol 1995, 26:53-61.

23. Uhal BD, Joshi I, True AL, Mundle S, Raza A, Pardo A, Selman M: Fibroblasts isolated after fibrotic lung injury induce apoptosis of alveolar epithelial cells in vitro. Am J Physiol 1995, 269: L819-828.

24. Wang R, Ramos C, Joshi I, Zagariya A, Pardo A, Selman M, Uhal $B$ : Human lung myofibroblast-derived inducers of alveolar epithelial apoptosis identified as angiotensin peptides. $A m \mathrm{~J}$ Physiol 1999, 277: L1158-L1164.

25. Uhal BD, Joshi I, Hughes WF, Ramos C, Pardo A, Selman M: Alveolar epithelial cell death adjacent to underlying myofibroblasts in advanced fibrotic human lung. Am J Physiol 1998, 275:L1192-L1199.

26. Barbas-Filho JV, Ferreira MA, Sesso A, Kairalla RA, Carvalho CR, Capelozzi VL: Evidence of type II pneumocyte apoptosis in the pathogenesis of idiopathic pulmonary fibrosis (IFP)/usual interstitial pneumonia (UIP). J Clin Pathol 2001, 54:132-138.

27. Kuwano K, Miyazaki H, Hagimoto N, Kawasaki M, Fujita M, Kunitake R, Kaneko Y, Hara N: The involvement of Fas-Fas ligand pathway in fibrosing lung diseases. $\mathrm{Am} J$ Respir Cell $\mathrm{Mol} \mathrm{BiO}$ 1999, 20:53-60.

28. Maeyama T, Kuwano K, Kawasaki M, Kunitake $R$, Hagimoto $N$ Matsuba T, Yoshimi M, Inoshima I, Yoshida K, Hara N: Upregulation of Fas-signalling molecules in lung epithelial cells from patients with idiopathic pulmonary fibrosis. Eur Respir J 2001, 17:180-189.

29. Ehrlich HP, Desmouliere A, Diegelmann RF, Cohen IK, Compton CC, Garner WL, Kapanci Y, Gabbiani G: Morphological and immunochemical differences between keloid and hypertrophic scar. Am J Pathol 1994, 145:105-113.

30. Chodon T, Sugihara T, Igawa HH, Funayama E, Furukawa $\mathrm{H}$ : Keloid-derived fibroblasts are refractory to Fas-mediated apoptosis and neutralization of autocrine transforming growth factor-beta 1 can abrogate this resistance. Am J Pathol 2000, 157:1661-1669.

31. Luo S, Benathan M, Raffoul W, Panizzon RG, Egloff DV: Abnormal balance between proliferation and apoptotic cell death in fibroblasts derived from keloid lesions. Plast Reconstr Surg 2001, 107:87-96

32. Lappi-Blanco E, Soini Y, Paakko P: Apoptotic activity is increased in the newly formed fibromyxoid connective tissue in bronchiolitis obliterans organizing pneumonia. Lung 1999, 177:367-376.

33. Ramos C, Montaño M, García-Alvarez J, Ruiz V, Uhal BD, Selman $M$, Pardo A: Fibroblasts from idiopathic pulmonary fibrosis and normal lungs differ in growth rate, apoptosis, and TIMPs expresion. Am J Respir Cell Mol Biol 2001, 24:591-598.

34. Jelaska A, Korn JH: Role of apoptosis and transforming growth factor beta 1 in fibroblast selection and activation in systemic sclerosis. Arthritis Rheum 2000, 43:2230-2239.

35. Jordana M, Schulman J, McSharry C, Irving LB, Newhouse MT, Jordana G, Gauldie J: Heterogeneous proliferative characteristics of human adult lung fibroblast lines and clonally derived fibroblasts from control and fibrotic tissue. Am Rev Respir Dis 1988, 137:579-584.

36. Raghu G, Chen YY, Rusch V, Rabinovitch PS. Differential proliferation of fibroblasts cultured from normal and fibrotic human Lung. Am Rev Respir Dis 1988, 138:703-708.

37. Legrand C, Polette M, Tournier JM, de Bentzmann S, Huet E, Monteau M, Birembaut P: UPa/plasmin system-mediated 
MMP-9 activation is implicated in bronchial epithelial cell migration. Exp Cell Res 2001, 264:326-336.

38. Davis G, Pintar Allen K, Salazar R, Maxwell S: Matrix metalloproteinase-1 and -9 activation by plasmin regulates a novel endothelial cell-mediated mechanism of collagen gel contraction and capillary tube regression in three-dimensional collagen matrices. J Cell Sci 2001, 114:917-930.

39. Kotani I, Sato A, Hayakawa H, Urano T, Takada Y, Takada A: Increased procoagulant and antifibrinolytic activities in the lungs with idiopathic pulmonary fibrosis. Thromb Res 1995, 77:493-504.

40. Imokawa S, Sato A, Hayakawa H, Kotani M, Urano T, Takada A: Tissue factor expression and fibrin deposition in the lungs of patients with idiopathic pulmonary fibrosis and systemic sclerosis. Am J Respir Crit Care Med 1997, 156:631-636.

41. Fujii $M$, Hayakawa $H$, Urano $T$ : Relevance of tissue factor and tissue factor pathway inhibitor for hypercoagulable state in the lungs of patients with idiopathic pulmonary fibrosis. Thromb Res 2000, 99:111-117.

42. Saito $Y$, Suzuki E, Moriyama H, Terada M, Hasegawa $T$, Ooi $H$, Kobayashi Y, Tsuchiya T, Geivo F: Decreased ability to generate plasmin in lung tissue of usual interstitial pneumonia compared with bronchiolitis obliterans organizing pneumonia. Am J Respir Crit Care Med 2000, 161:A826.

43. Higgins PJ, Slack JK Diegelmann RF, Staiano-Coico L: Differential regulation of PAl-1 gene expression in human fibroblasts predisposed to a fibrotic phenotype. Exp Cell Res 1999, 248: 634-642.

44. Hayashi T, Stetler-Stevenson WG, Fleming MV, Fishback N, Koss MN, Liotta LA, Ferrans VJ, Travis WD: Immunohistochemical study of metalloproteinases and their tissue inhibitors in the lungs of patients with diffuse alveolar damage and idiopathic pulmonary fibrosis. Am J Pathol 1996, 149:1241-1256.

45. Fukuda Y, Ishizaki M, Kudoh S, Kitaichi M, Yamanaka N: Localization of matrix metalloproteinases $-1,-2$, and -9 , and tissue inhibitor of metalloproteinase-2 in interstitial lung diseases. Lab Invest 1998, 78:687-698.

46. Selman M, Ruiz V, Cabrera S, Segura L, Ramírez R, Barrios R, Pardo A: TIMP 1, 2, 3 and 4 in idiopathic pulmonary fibrosis. A prevailing non-degradative lung microenvironment? $\mathrm{Am} J$ Physiol 2000, 279:L562-L574.

47. Zhang K, Rekhter MD, Gordon D, Phan SH: Myofibroblasts and their role in lung collagen gene expression during pulmonary fibrosis. Am J Pathol 1994, 145:114-125.

48. Parks WC, Sudbeck BD, Doyle GR, Saariahlo-Kere UK: Matrix metalloproteinases in tissue repair. In Matrix Metalloproteinases. Edited by Parks WC, Mecham RP. San Diego: Academic Press; 1998:263-297. 